\title{
Efficacy of the Erector Spinae Plane Block for Lumbar Spinal Surgery: A Retrospective Study
}

\author{
Hironobu Ueshima ${ }^{1}$, Mayumi Inagaki ${ }^{1}$, Tomoaki Toyone ${ }^{2}$, Hiroshi Otake ${ }^{1}$ \\ ${ }^{1}$ Department of Anesthesiology, Showa University Hospital, Tokyo, Japan \\ ${ }^{2}$ Department of Orthopedics, Showa University Hospital, Tokyo, Japan
}

\section{Study Design: A retrospective study.}

Purpose: The first research on the erector spinae plane (ESP) block was published in 2016. To our knowledge, no cohort studies or randomized controlled trials of the ESP block were performed in 2016 and 2017.

Overview of the Literature: This study retrospectively investigated the efficacy of the ESP block in pain management after lumbar spinal surgery.

Methods: Patients who underwent lumbar spinal surgery in 2017 were enrolled in the study. Those who underwent secondary surgery with local anesthesia other than the ESP block were excluded. The primary outcome was the Numerical Rating Scale (NRS) pain score at various time points until the morning of postoperative day 2. The secondary outcomes were the amount of intravenous fentanyl administered during the first 24 hours following the surgery and the number of patients with complaints of complications such as nausea and vomiting until the morning of postoperative day 2 .

Results: The data of 41 patients undergoing lumbar spinal surgery were retrospectively analyzed. Of these, 23 received only general anesthesia ( $G$ group), whereas the other 18 patients received the ESP block in addition to general anesthesia (E group). The NRS pain scores and the amount of fentanyl administered were lower in the $\mathrm{G}$ group than in the $\mathrm{E}$ group at all measured time points (all data were less than $p<0.05)$. There was no significant difference in the incidence of complications between the two groups $(p=0.11)$. Conclusions: The ESP block provides effective postoperative analgesic effect for 24 hours in patients undergoing lumbar spinal surgery.

Keywords: Spinal nerve; Lumbar verrebrae; Pain management

\section{Introduction}

The erector spinae plane (ESP) block, first published in 2016 , has been performed in various surgeries $[1,2]$. The majority of the papers that discuss the ESP block are case reports. To our knowledge, no cohort studies or randomized controlled trials have been performed in 2016 and 2017 on the ESP block technique [3-5]. This study is the first to retrospectively investigate the efficacy of the ESP block in pain management after lumbar spinal surgery.

\section{Materials and Methods}

This retrospective study was approved by the Showa University Hospital Institutional Review Board (approval no., 2583) and was registered at the University Hospital Medi-

Received May 17, 2018; Revised Jul 20, 2018; Accepted Aug 13, 2018

Corresponding author: Hironobu Ueshima

Department of Anesthesiology, Showa University Hospital, 1-5-8 Hatanodai Shinagawa-ku, Tokyo, 142-8666 Japan

Tel: +81-03-3784-8875, Fax: +81-03-3784-8357, E-mail: ueshimhi@yahoo.co.jp 
cal Information Network (UMIN ID number 000032418). We included patients who underwent lumbar microendoscopic surgery in 2017. The enrolled subjects had access to intravenous patient-controlled analgesia that comprised $0.5 \mu \mathrm{g} \mathrm{kg}^{-1} \mathrm{~h}^{-1}$ continuous fentanyl administration and $0.5 \mu \mathrm{g} \mathrm{kg}^{-1}$ fentanyl bolus infusion when requested by the patients. The bolus was permitted at intervals of $\geq 10$ minutes. The exclusion criteria included conversion to open surgery, secondary surgery, and regional anesthesia other than an ESP block. Pre- and perioperative data regarding age, height, weight, American Society of Anesthesiologist classification, surgery duration, anesthesia duration, and amount of fentanyl and remifentanil administered during the perioperative period were collected. All the patients were moved to the high-care unit in our hospital within 48 hours after the surgery. The primary outcome measurement was the Numerical Rating Scale (NRS) pain score (a scale of $0-10$, where $0=$ no pain and $10=$ worst pain) at various time points until the morning of postoperative day 2. Each patient's pain level was measured by two nurses at $1,2,4,6,12$, and 24 hours postoperatively and on the morning of postoperative day 2 . The secondary outcome measures were the amount of intravenous fentanyl bolus administered during the first 24 hours after surgery and the number of patients who reported complications such as nausea and vomiting until the morning of postoperative day 2. All patients regularly took oral celecoxib (200 mg twice daily) for 2 days orally. If a patient was unable to control the pain, an attending anesthesiologist was consulted, and if necessary, the prescription was changed

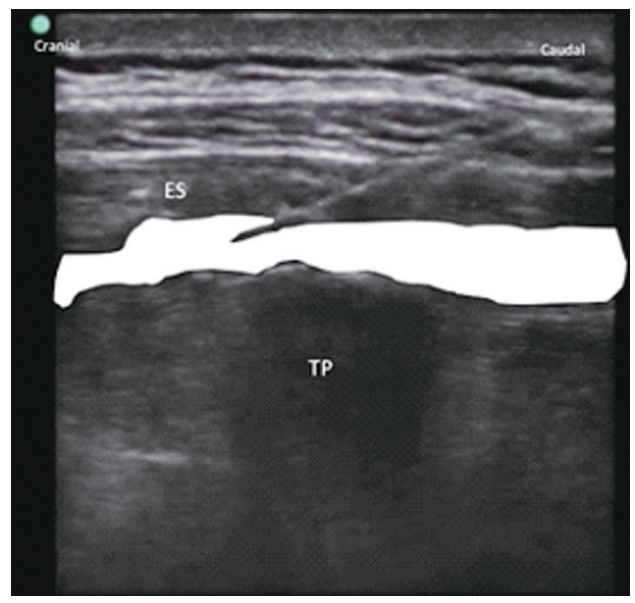

Fig. 1. Ultrasound image of local anesthetic (white frame) after erector spinae plane block. Local anesthetic spread to the deep surface of the erector plane muscle (ES). TP, transverse process; ES, erector spinae. from continuous administration to a different dose. Patients who reported nausea and vomiting were injected with $10 \mathrm{mg}$ of intravenous metoclopramide.

\section{Erector spinae plane block technique}

A high-frequency linear ultrasound transducer was sagittally placed against the target vertebral level in the prone position and moved in approximately $3-\mathrm{cm}$ lateral to the spinous process $[1,2]$. The erector spinae muscle and transverse muscle were then identified, and a needle was advanced through the interfascial plane between the erector spinae and the underlying transverse process; thereafter, the local anesthetic was administered into the space (Fig. 1). The bilateral ESP blocks were performed by injecting $40 \mathrm{~mL}$ of $0.375 \%$ levobupivacaine $(20 \mathrm{~mL}$ into each side) into the fascial plane between the deep surface of the erector spinae muscle and the transverse processes of the lumbar vertebrae for pain management after lumbar spinal surgery.

\section{Statistical analyses}

Statistical analyses were performed using JMP ver. 12.0 (SAS Institute Inc., Cary, NC, USA). Pain scores and the amount of fentanyl bolus administered were analyzed using the Mann-Whitney $U$-test. The number of patients who reported complications such as nausea and vomiting was analyzed using Fisher's exact test. Data are expressed as median (interquartile range) $(\mu \mathrm{g})$ values. The level of significance for both the tests was set at $p<0.05$.

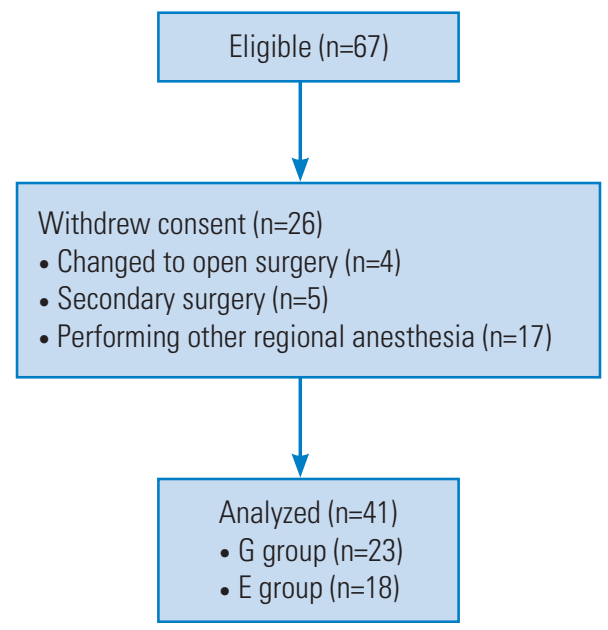

Fig. 2. Flow chart of the study. 
Table 1. Patients demographics data

\begin{tabular}{lcc} 
Characteristic & G group $(\mathrm{n}=23)$ & E group (n=18) \\
Age $(\mathrm{yr})$ & $70(53.5-75)$ & $68(54-74.5)$ \\
Sex (male:female) & $12: 11$ & $9: 9$ \\
\hline Body mass index $\left(\mathrm{kg} / \mathrm{m}^{2}\right)$ & $24(21.9-27.2)$ & $23.5(20.2-25.6)$ \\
American Society of Anesthesiologists classification (I/II/III) & $4 / 19 / 0$ & $3 / 15 / 0$ \\
\hline Operation time (min) & $155(127.5-180)$ & $115(78.8-150)$ \\
Anesthesia time (min) & $210(192.5-245)$ & $147.5(115-186.3)$ \\
\hline
\end{tabular}

Values are presented as median (interquartile range) or number.

Table 2. The contents of surgical procedure

\begin{tabular}{lcc}
$\begin{array}{l}\text { At the site of lumbar } \\
\text { laminoplasty }\end{array}$ & $\mathrm{G}$ group $(n=23)$ & E group $(n=18)$ \\
$L 1 / 2$ & 0 & 0 \\
$L 2 / 3$ & 0 & 0 \\
$L 3 / 4$ & 4 & 4 \\
$L 4 / 5$ & 6 & 5 \\
$L 5 / S 1$ & 2 & 0 \\
$L 1 / 2 / 3$ & 0 & 0 \\
$L 2 / 3 / 4$ & 7 & 2 \\
$L 3 / 4 / 5$ & 3 & 6 \\
$L 4 / 5 / S 1$ & 1 & 1 \\
\hline
\end{tabular}

\section{Results}

In total, 67 patients were analyzed; of these, 26 were excluded. Of the remaining 41 patients, 23 received only general anesthesia ( $G$ group), whereas the other 18 received the ESP block in addition to general anesthesia (E group) (Fig. 2). Patient demographic data are reported in Table 1, and Table 2 lists the surgical procedures as per the treatment groups. The ESP block was performed by anesthesiologists who were skilled in ultrasound-guided nerve block. The NRS pain scores in the E group were lower at $1,2,4,6,12$, and 24 hours, as well as on the morning of postoperative day 2, as compared with those in the $G$ group (comparisons at all measured time points were $p<0.05)$ (Fig. 3). The amount (40 [40-60] $\mu \mathrm{g}$ ) of fentanyl bolus administration in the E group was lower than that $(100[80-100] \mu \mathrm{g})$ in the G group during the first 24 hours postoperatively $(p<0.05)$. Six patients in the $\mathrm{G}$ group and one patient in the E group experienced complications; however, the two groups did not exhibit significant differences in the incidence of complications such as nausea

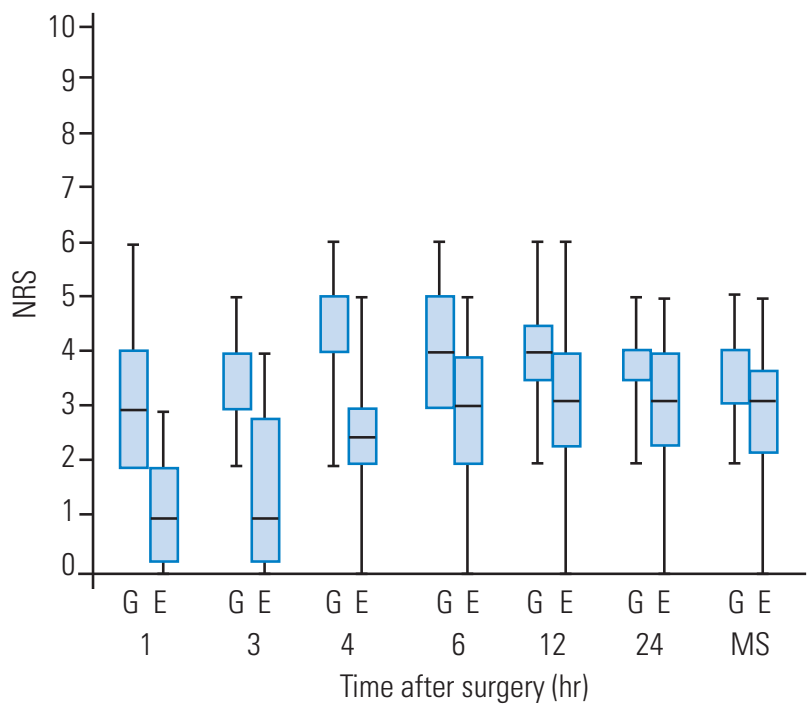

Fig. 3. The NRS pain scores at 1, 2, 4, 6, 12, and 24 hours and on the MS. NRS, Numerical Rating Scale; G, G group; E, E group; MS, morning of postoperative day 2 .

Table 3. The amount of fentanyl bolus administration intravenously during the first 24 hours after surgery and the number of patients complaining of complications, such as nausea and vomiting, until the morning in the second postoperative day

\begin{tabular}{lcccc} 
& G group & E group & $p$-value \\
$\begin{array}{lccc}\text { Amount of fentanyl bolus } \\
\text { administration }(\mu \mathrm{g})\end{array}$ & $100(80-100)$ & $40(40-60)$ & $<0.05$ \\
No. of complications & 6 & 1 & 0.11 \\
\hline
\end{tabular}

Values are presented as median (interquartile range) or number.

and vomiting $(p=0.11)$ (Table 3$)$.

\section{Discussion}

These results indicate that the ESP block was able to exert an effective analgesic effect until the morning of postoperative day 2 following lumbar laminoplasty. Thus, the ESP 
block affected the dorsal rami of the lumbar nerves. This study reported similar results as another cadaveric study [6]. Compared with other peripheral nerve blocks, such as epidural anesthesia and paravertebral nerve block, the ESP block is safer because it is a sensory nerve block [7-9]. Moreover, the ESP block reportedly relieves perioperative pain over a wide region and is therefore an effective analgesic for various surgeries [1-5,10].

The present study has limitations. The range of blocked nerve could not be measured; therefore, the analgesic effect of the ESP block in lumbar spine surgery was unclear. Moreover, although the effective analgesic time of levobupivacaine is generally believed to be within 12 hours, this study showed that the ESP block using levobupivacaine provided effective pain relief for the first 24 hours after the surgery. The underlying reason for this prolonged effect of the ESP block remains unclear. The systemic effect for levobupivacaine was perhaps affected.

Further, this study only focused on the first 48 hours after surgery. However, we are unaware of the efficacy of the ESP block in reducing chronic pain. A prospective study should be considered in the near future to clarify these issues.

\section{Conclusions}

The ESP block for patients undergoing lumbar laminoplasty provides more effective analgesia that persists until the morning of postoperative day 2 .

\section{Conflict of Interest}

No potential conflict of interest relevant to this article was reported.

\section{Author Contributions}

Study design and planning: HU, HO; study conduct: all authors; data analysis: $\mathrm{HU}, \mathrm{HO}$; writing paper: $\mathrm{HU}$; and revising paper: all authors.

\section{References}

1. Forero M, Adhikary SD, Lopez H, Tsui C, Chin KJ. The erector spinae plane block: a novel analgesic technique in thoracic neuropathic pain. Reg Anesth Pain Med 2016;41:621-7.

2. Chin KJ, Malhas L, Perlas A. The erector spinae plane block provides visceral abdominal analgesia in bariatric surgery: a report of 3 cases. Reg Anesth Pain Med 2017;42:372-6.

3. Ueshima H, Otake H. Clinical experiences of erector spinae plane block for children. J Clin Anesth 2018;44:41.

4. Munoz F, Cubillos J, Bonilla AJ, Chin KJ. Erector spinae plane block for postoperative analgesia in pediatric oncological thoracic surgery. Can J Anaesth 2017;64:880-2.

5. Aksu C, Gurkan Y. Ultrasound guided erector spinae block for postoperative analgesia in pediatric nephrectomy surgeries. J Clin Anesth 2018;45:35-6.

6. Ivanusic J, Konishi Y, Barrington MJ. A cadaveric study investigating the mechanism of action of erector spinae blockade. Reg Anesth Pain Med 2018;43:567-71.

7. Kulhari S, Bharti N, Bala I, Arora S, Singh G. Efficacy of pectoral nerve block versus thoracic paravertebral block for postoperative analgesia after radical mastectomy: a randomized controlled trial. Br J Anaesth 2016;117:382-6.

8. El-Boghdadly K, Madjdpour C, Chin KJ. Thoracic paravertebral blocks in abdominal surgery: a systematic review of randomized controlled trials. Br J Anaesth 2016;117:297-308.

9. Andreae MH, Andreae DA. Regional anaesthesia to prevent chronic pain after surgery: a Cochrane systematic review and meta-analysis. Br J Anaesth 2013;111:711-20.

10. De la Cuadra-Fontaine JC, Concha M, Vuletin F, Arancibia H. Continuous erector spinae plane block for thoracic surgery in a pediatric patient. Paediatr Anaesth 2018;28:74-5. 